\title{
Indicadores empíricos para avaliação da condição de saúde da pessoa idosa pelo
} Enfermeiro na Atenção Básica

\author{
Empirical indicators for Nurses' health condition assessment in the Primary Care \\ Indicadores empíricos para la evaluación del estado de salud de las personas mayores por \\ Enfermeras de Cuidados Básicos
}

Recebido: 18/02/2021 | Revisado: 25/02/2021 | Aceito: 01/03/2021 | Publicado: 07/03/2021

\author{
Renata Maria Mota Wanderley \\ ORCID: https://orcid.org/ 0000-0003-4856-0886 \\ Universidade Federal de Pernambuco, Brasil \\ E-mail: renata-mota@hotmail.com \\ Divany Guedes Pereira da Cunha \\ ORCID: https://orcid.org/0000-0003-0114-7840 \\ Universidade Federal da Paraíba, Brasil \\ E-mail: divany.pereira@hotmail.com \\ Greicy Kelly Gouveia Dias Bittencourt \\ ORCID: https://orcid.org/0000-0001-5287-8171 \\ Universidade Federal da Paraíba, Brasil \\ E-mail: greicykel@gmail.com \\ Ana Karênina de Freitas Jordão do Amaral \\ ORCID: https://orcid.org/0000-0002-7470-7717 \\ Universidade Federal da Paraíba, Brasil \\ E-mail: akfjafono@hotmail.com \\ Antonia Oliveira Silva \\ ORCID: https://orcid.org/0000-0001-7758-2035 \\ Universidade Federal da Paraíba, Brasil \\ E-mail: alfaleda2@gmail.com \\ Tássia Daniele Lima Almeida \\ ORCID: https://orcid.org/0000-0002-7164-8096 \\ Universidade Federal de Pernambuco, Brasil \\ E-mail: tassia.daniele@ hotmail.com \\ Sabrina Emylle Torres Fernandes \\ ORCID: https://orcid.org/0000-0002-4703-4895 \\ Universidade Federal de Pernambuco, Brasil \\ E-mail: sabrinaemylle.torres@gmail.com
}

\begin{abstract}
Resumo
Objetivo: Identificar indicadores empíricos na literatura, a partir das necessidades humanas básicas da pessoa idosa; e confirmar a utilidade de indicadores empíricos para avaliação da condição de saúde da pessoa idosa na atenção básica pelo enfermeiro. Método: Trata-se de um estudo exploratório descritivo com abordagem quantitativa. Foi realizado em duas etapas: 1) Identificação dos indicadores empíricos, na literatura, a partir das necessidades humanas básicas da pessoa idosa; e 2) Validação dos indicadores empíricos pelos enfermeiros participantes da pesquisa, calculando-se o Índice de Validade de Conteúdo, considerando-se válidos os indicadores que obtiveram IVC $\geq 0.80$. Resultados: Foram identificados 316 indicadores empíricos na literatura, destes, 255 obtiveram IVC $\geq 0.80$. Dos 255 indicadores validados, 205 foram das necessidades psicobiológicas, 47 das necessidades psicossociais e 3 das necessidades psicoespirituais. Conclusão: O idoso apresenta características específicas que o diferencia de outros tipos de indivíduos. Acredita-se que os achados dessa pesquisa venham a contribuir com a implantação da sistematização da assistência de enfermagem aos idosos, tendo em vista o planejamento de ações de saúde com atenção diferenciada e sistematizada para essa população na atenção básica.
\end{abstract}

Palavras-chave: Enfermagem; Processo de enfermagem; Atenção primária à saúde; Saúde do idoso.

\begin{abstract}
Objective: Identify empirical indicators in the literature, based on the basic human needs of the elderly; and confirm the usefulness of empirical indicators to assess the health condition of the elderly in primary care by nurses. Method: This is an exploratory, descriptive study with a quantitative approach. It was carried out in two stages: 1) Identification of empirical indicators, in the literature, based on the basic human needs of the elderly person; and 2) Validation of the empirical indicators by the nurses participating in the research, calculating the Content Validity Index, considering the indicators that obtained CVI $\geq 0.80$ to be valid. Results: 316 empirical indicators were
\end{abstract}


identified in the literature, of these, 255 obtained CVI $\geq 0.80$. Of the 255 validated indicators, 205 were for psychobiological needs, 47 for psychosocial needs and 3 for psycho-spiritual needs. Conclusion: The elderly has specific characteristics that differentiate them from other types of individuals. It is believed that the findings of this research will contribute to the implementation of the systematization of nursing care for the elderly, with a view to planning health actions with differentiated and systematized care for this population in primary care.

Keywords: Nursing; Nursing process; Primary health care; Health of the elderly.

\section{Resumen}

Objetivo: Identificar indicadores empíricos en la literatura, basados en las necesidades humanas básicas del anciano; y confirmar la utilidad de los indicadores empíricos para evaluar el estado de salud de los ancianos en la atención primaria por enfermeras. Método: Se trata de un estudio exploratorio, descriptivo con enfoque cuantitativo. Se llevó a cabo en dos etapas: 1) Identificación de indicadores empíricos, en la literatura, basados en las necesidades humanas básicas del anciano; y 2) Validación de los indicadores empíricos por parte de los enfermeros participantes de la investigación, calculando el Índice de Validez de Contenido, considerando válidos los indicadores que obtuvieron CVI $\geq 0,80$. Resultados: se identificaron 316 indicadores empíricos en la literatura, de estos, 255 obtuvieron IVC $\geq 0,80$. De los 255 indicadores validados, 205 fueron para necesidades psicobiológicas, 47 para necesidades psicosociales y 3 para necesidades psico-espirituales. Conclusión: el anciano tiene características específicas que lo diferencian de otro tipo de individuos. Se cree que los hallazgos de esta investigación contribuirán a la implementación de la sistematización de la atención de enfermería al adulto mayor, con miras a planificar acciones de salud con atención diferenciada y sistematizada para esta población en atención primaria.

Palabras clave: Enfermería; Proceso de enfermería; Primeros auxilios; Salud de las personas mayores.

\section{Introdução}

A Enfermagem experimenta um processo de intensa evolução ao longo dos anos, o que interfere diretamente no reconhecimento dessa categoria profissional. Diante disso, a Enfermagem, antes conhecida empiricamente se transformou em práticas embasadas cientificamente, possibilitando a promoção à saúde aos indivíduos. Assim, foram criadas teorias de Enfermagem com o objetivo de aproximar-se da complexidade e favorecer novos conhecimentos que orientam a prática cotidiana do profissional enfermeiro (Pinto et al., 2017).

A teoria de enfermagem pioneira no Brasil foi criada em 1970, a Teoria das Necessidades Humanas Básicas, proposto por Wanda Horta. Para atender as demandas do ser humano, é essencial fomentar ações que utilizem recursos científicos. Então, surgiu o processo de enfermagem como sendo “a dinâmica das ações sistemáticas e inter-relacionadas, visando a assistência integral aos indivíduos”. A elaboração de um plano terapêutico guiado por teorias e ciência proporciona cuidados preventivos e de reabilitação, o que resulta na aproximação do profissional enfermeiro ao paciente, além de proporcionar uma assistência pautada em evidências clínicas (Horta, 2005 \& Jacon et al., 2020).

O modelo proposto por Wanda Horta se referiu ao processo de cuidado e que se dividia em seis fases interrelacionadas contendo: Histórico de enfermagem; Diagnóstico de enfermagem; Plano assistencial; Prescrição de enfermagem e Evolução de enfermagem. Tal modelo teve o objetivo de organizar o gerenciamento do cuidado, embasado em metodologias científicas e não unicamente no empirismo. Assim, os dados coletados, levaria à identificação de um problema, as condutas de enfermagem seguiriam um planejamento dos cuidados e, após sua implementação, evoluiria para uma avaliação de resultados e consequente novo planejamento. Todas estas fases deveriam ser registradas em prontuário do paciente pelo corpo de enfermagem (Silva, 2014).

A realização da consulta de enfermagem tem seu aporte legal amparado na Lei do Exercício Profissional da Enfermagem, $n^{\circ} 7.498 / 86$, que a legitima como sendo uma atividade prioritária do enfermeiro (Brasil, 1986). A Resolução ${ }^{\circ}$ 159/93, do Conselho Federal de Enfermagem (COFEN), enfatiza que a consulta de enfermagem utiliza contribui para a promoção, prevenção e proteção da saúde, recuperação e reabilitação do indivíduo, família e comunidade (Conselho Federal de Enfermagem, 1993). 
A consulta de enfermagem necessita ser repercutida na prática diária do enfermeiro, sendo uma das ações de grande importância do profissional enfermeiro na Estratégia Saúde da Família (ESF). Nesse sentido, o papel do profissional enfermeiro pode oportunizar um vínculo positivo com a população de seu território, prestando um cuidado efetivo ao estabelecer o diálogo e resolução das demandas reais entre a comunidade e a unidade básica de saúde (Ferreira \& Abrahão, 2020).

O papel do enfermeiro na ESF tem possibilitado a assistência de saúde, assim, entre os principais cuidados ao idoso, destaca-se o apoio a população idosa no que tange a educação em saúde sobre o processo saúde-doença. Portanto, a prestação de cuidados de enfermagem aos idosos contribuiu na redução e prevenção de danos estruturais e funcionais (Martins et al., 2020).

Apesar de uma grande proporção de enfermeiros não registrarem a assistência prestada de forma sistematizada, é essencial organizar esse cuidado através da implementação do Processo de Enfermagem. Nesse interim, a operacionalização e o registro do Processo de Enfermagem contribuem na consolidação da atenção à saúde da população, assim como, promove visibilidade e o reconhecimento profissional (Siqueira, 2020).

Logo, levando em conta que o Processo de Enfermagem é formado por etapas, e que a primeira - histórico de enfermagem - é de suma importância para o desenrolar das outras etapas desse processo, é necessário dispor de um instrumento que possa solucionar os problemas prioritários dos indivíduos envolvidos. Dessa forma, aliar o conhecimento científico com a sensibilidade crítica do profissional é de grande valia (Piccinini, Costa \& Pissaia, 2018).

Com isso, destaca-se a necessidade da pesquisadora em realizar uma revisão da literatura sobre o cuidado ao idoso na atenção básica, anteriormente à constituição de um instrumento, permitindo a ampliação do conhecimento e proporcionando um roteiro sistematizado para o levantamento de sinais e sintomas (indicadores empíricos) do ser humano que evidenciassem necessidades humanas básicas acometidas. Os indicadores revelam situações consideradas habituais ou esperadas, sendo a identificação destas fundamentais para adequar um planejamento efetivo ao indivíduo através da consulta de enfermagem (Cheloni, Silva \& Souza, 2020).

Logo, buscou-se, neste estudo, responder à seguinte questão norteadora: Que indicadores empíricos representam as necessidades de saúde da pessoa idosa a serem consideradas durante avaliação da sua condição de saúde pelo enfermeiro? Nessa perspectiva, este estudo teve como objetivos: identificar indicadores empíricos na literatura, a partir das necessidades humanas básicas da pessoa idosa; e confirmar a utilidade de indicadores empíricos para avaliação da condição de saúde da pessoa idosa na atenção básica pelo enfermeiro.

\section{Metodologia}

Trata-se de um estudo exploratório descritivo com abordagem quantitativa. Nesse interim, o estudo quantitativo possibilita interpretar e compreender os fenômenos que acontecem nas diversas áreas do saber (Pereira et al., 2018). Além disso, o método quantitativo tem seu foco voltado na objetividade e confiabilidade do objeto de estudo (Silva, Russo \& Oliveira, 2018), contribuindo para a efetivação da referida pesquisa. Assim, para a realização do estudo foram seguidas duas etapas: 1) Identificação dos indicadores empíricos, na literatura, a partir das necessidades humanas básicas da pessoa idosa; e 2) Validação dos indicadores empíricos pelos enfermeiros participantes da pesquisa.

Inicialmente consistiu-se uma revisão integrativa da literatura e pesquisa bibliográfica através de livros, teses, dissertações e manuais, onde foi feito um levantamento sobre as necessidades humanas básicas e os cuidados com o idoso na atenção básica. Foi realizada busca com fichamento de artigos publicados em periódicos indexados nas seguintes bases de dados: Bases de Dados de Enfermagem (BDENF), Literatura Latino-Americana e do Caribe em Ciências da Saúde (LILACS), Medical Literature Analysis and Retrieval System Online/Biblioteca Virtual em Saúde (MEDLINE/BVS) e Scientific Eletronic 
Library Online (SciELO), utilizando-se os seguintes descritores catalogados pelo DeCS: idoso, processos de enfermagem, nível de saúde e atenção primária a saúde.

Os indicadores empíricos são proposições experimentais usados para mensurar e fornecer evidências sobre a conceitualização de uma teoria (Fawcet, 2013). Neste estudo, consideraram-se indicadores empíricos as revelações das necessidades humanas básicas alteradas na pessoa idosa.

Para elencar os indicadores empíricos encontrados, empregou-se como referencial teórico a Teoria das Necessidades Humanas Básicas de Wanda Aguiar Horta, sendo estas em subdivididas em três pontos: psicobiológicas, psicossociais e psicoespirituais (Horta, 2005).

A segunda etapa do estudo foi constituída pela validação dos indicadores empíricos pelos enfermeiros participantes da pesquisa. Os participadores da pesquisa foram 28 enfermeiros atuantes nas Unidades de Saúde da Família do Município de Igarassu-PE. Os indicadores descobertos na literatura foram listados e categorizados em um instrumento construído para que os participantes avaliassem a relevância de cada indicador encontrado. Além disso, tal instrumento acompanhou uma carta de esclarecimento, com o objetivo de nortear a avaliação e o julgamento dos indicadores empíricos pelos enfermeiros.

A validação ocorreu através do cálculo do Índice de Validade de Conteúdo (IVC) para cada indicador empírico por intermédio da fórmula IVC = número de respostas 3 ou 4 / número total de respostas. Este método emprega uma escala com pontuação de um a quatro. Para avaliar a relevância/representatividade, as respostas podem incluir: 1 = não útil, 2 = pouco útil, 3 = bastante útil, $4=$ muito útil (Alexandre \& Coluci, 2011).

Foram considerados validados os indicadores empíricos que alcançaram um índice de validade de conteúdo (IVC) $\geq$ 0.80. Após a validação dos indicadores empíricos foi produzido um novo instrumento contendo apenas os indicadores validados (Quadro 1).

Figura 1: Etapas metodológicas do estudo. Igarassu-PE, Brasil, 2017.

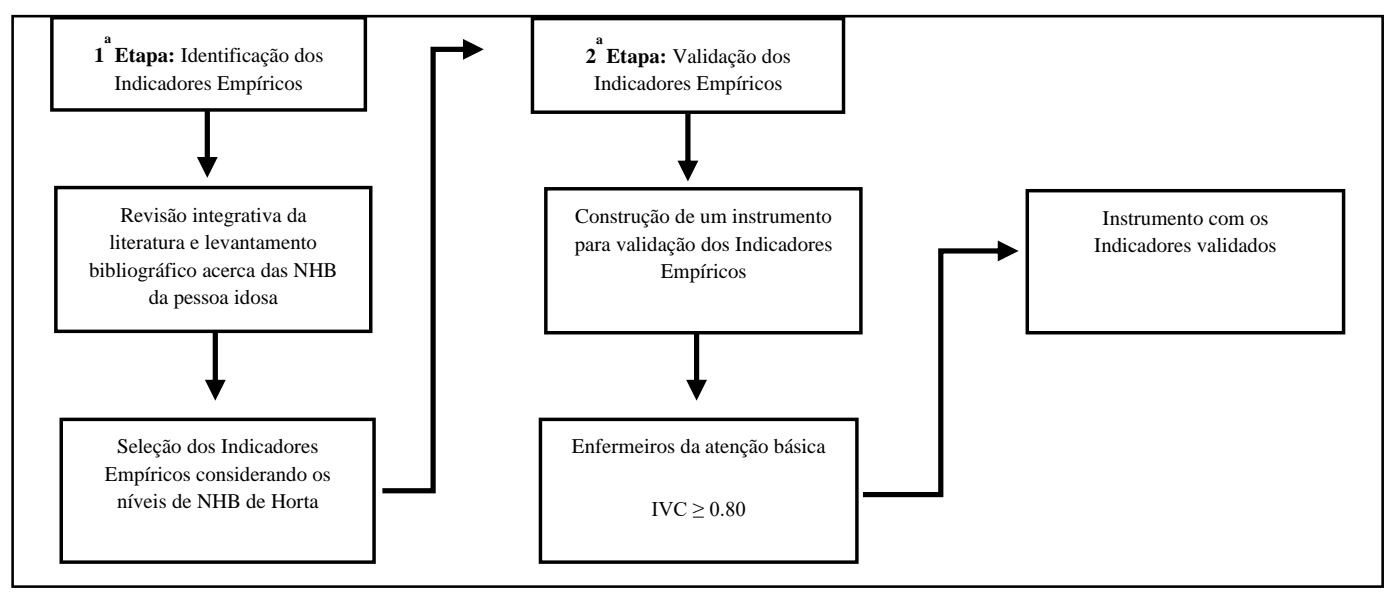

Fonte: Autoria da pesquisa.

Com o intuito de conhecer os indicadores analisados pelos enfermeiros, os dados constantes nos instrumentos foram compilados em planilhas do Excel for Windows, e aqueles que apresentaram IVC $\geq 0.80$ foram listados para compor um instrumento de avaliação da condição de saúde da pessoa idosa na atenção básica. Para a análise dos dados foi utilizada a estatística descritiva por meio da frequência absoluta e percentual e realizado o teste de proporções objetivando enfatizar os dados mais relevantes. 


\section{Resultados}

Na primeira etapa do estudo foram identificados 316 (100\%) indicadores empíricos, sendo 243 (76,9\%) indicadores das necessidades psicobiológicas, $67(21,2 \%)$ pertencentes às necessidades psicossociais e $6(1,9 \%)$ às necessidades psicoespirituais (Tabela 1).

Tabela 1: Indicadores empíricos das Necessidades Humanas Básicas em Idosos na Atenção Básica. Igarassu-PE, Brasil, 2017.

\begin{tabular}{lcc}
\hline Indicadores Empíricos & N & \% \\
\hline NHB Psicobiológicas & 243 & 76,9 \\
NHB Psicossociais & 67 & 21,2 \\
NHB Psicoespirituais & 06 & 1,9 \\
\hline \hline Total & $\mathbf{3 1 6}$ & $\mathbf{1 0 0 , 0}$ \\
\hline
\end{tabular}

Fonte: Dados da pesquisa.

A Tabela 1 apresenta a distribuição dos indicadores empíricos segundo os níveis de Necessidades Humanas Básicas (NHB) (Horta, 2005), observando-se que as necessidades fundamentais da natureza humana estiveram predominantemente relacionadas ao nível psicobiológico $(76,9 \%)$.

A segunda etapa foi marcada pelo julgamento dos indicadores empíricos levantados na literatura, que após serem analisados por um comitê de especialistas (experts na área do construto) subsidiou a constituição de um instrumento de coleta de dados para avaliação da condição de saúde da pessoa idosa na atenção básica. Do total de 316 indicadores empíricos identificados na literatura e analisados pelos enfermeiros, 255 (100\%) permaneceram com IVC $\geq 0.80$ e, desses indicadores validados, 205 (80,4\%) foram das necessidades psicobiológicas, 47 (18,43\%) das necessidades psicossociais e $3(1,17 \%)$ da necessidades psicoespirituais (Tabela 2 ).

Tabela 2: Indicadores empíricos das Necessidades Humanas Básicas em Idosos na Atenção Básica validados pelos enfermeiros das unidades básicas de saúde. Igarassu-PE, Brasil, 2017.

\begin{tabular}{lcc}
\hline Indicadores Empíricos & N & \% \\
\hline NHB Psicobiológicas & $\mathbf{2 0 5}$ & $\mathbf{8 0 , 4 0}$ \\
Nutrição & 23 & 9,01 \\
Eliminação & 19 & 7,45 \\
Integridade física e cutaneomucosa & 19 & 7,45 \\
Oxigenação & 17 & 6,70 \\
Percepção dos órgãos dos sentidos & 16 & 6,30 \\
Regulação neurológica & 14 & 5,50 \\
Hidratação & 13 & 5,10 \\
\hline
\end{tabular}




\begin{tabular}{|c|c|c|}
\hline Cuidado corporal & 12 & 4,70 \\
\hline Regulação térmica & 09 & 3,52 \\
\hline Regulação vascular & 08 & 3,13 \\
\hline Sexualidade & 06 & 2,35 \\
\hline Exercícios e atividades físicas & 05 & 1,96 \\
\hline Sono e repouso & 05 & 1,96 \\
\hline Regulação hormonal & 05 & 1,96 \\
\hline Abrigo & 05 & 1,96 \\
\hline Locomoção & 05 & 1,96 \\
\hline Ambiente & 04 & 1,56 \\
\hline Mecânica corporal & 04 & 1,56 \\
\hline Motilidade & 04 & 1,56 \\
\hline Terapêutica & 03 & 1,17 \\
\hline Regulação hidrossalina e eletrolítica & 03 & 1,17 \\
\hline Regulação imunológica & 03 & 1,17 \\
\hline Crescimento celular & 03 & 1,17 \\
\hline NHB Psicossociais & 47 & 18,43 \\
\hline Amor e aceitação & 10 & 3,92 \\
\hline Comunicação & 08 & 3,13 \\
\hline Segurança & 07 & 2,74 \\
\hline Autorrealização, autoestima e autoimagem & 06 & 2,35 \\
\hline Aprendizagem (educação à saúde) & 06 & 2,35 \\
\hline Liberdade e participação & 03 & 1,17 \\
\hline Espaço e atenção & 02 & 0,78 \\
\hline Recreação e lazer & 02 & 0,78 \\
\hline Socialibidade & 02 & 0,78 \\
\hline Criatividade & 01 & 0,39 \\
\hline
\end{tabular}

\section{NHB Psicoespirituais}

Religiosa ou teológica, ética ou de filosofia de vida $03 \quad 1,17$

\begin{tabular}{lcl}
\hline Total & 255 & 100,0
\end{tabular}

Fonte: Dados da pesquisa. 
A tabela 2 apresenta a relação dos indicadores empíricos que alcançaram IC $\geq 0.80$, podendo-se evidenciar o predomínio das manifestações das necessidades humanas básicas afetadas na pessoa idosa no nível psicobiológico, com destaque para as necessidades de nutrição $(9,01 \%)$, eliminação $(7,45 \%)$, integridade física e cutaneomucosa $(7,45 \%)$, oxigenação $(6,7 \%)$ e percepção dos órgãos dos sentidos (6,3\%). Além disso, termos como ansiedade, oligúria, depressão, paresia e parestesia se repetiam em mais de uma necessidade básica.

As características psicobiológicas, psicossociais e psicoespirituais associadas na Relação dos Indicadores Empíricos das Necessidades Humanas Básicas na pessoa idosa que alcançaram IVC $\geq 0.80$, de acordo com os enfermeiros da atenção básica, são apresentados a seguir (Quadro 1).

Quadro 1: Relação dos Indicadores Empíricos das Necessidades Humanas Básicas na pessoa idosa que alcançaram IVC $\geq$ 0.80, segundo enfermeiros da atenção da básica.

\begin{tabular}{|c|c|}
\hline $\begin{array}{l}\text { NECESSIDADES } \\
\text { HUMANAS } \\
\text { BÁSICAS }\end{array}$ & INDICADORES EMPÍRICOS \\
\hline \multicolumn{2}{|r|}{ PSICOBIOLÓGICAS } \\
\hline Oxigenação & $\begin{array}{l}\text { Ausculta pulmonar; bradipnéia; cansaço; cianose; dispneia; dor ao respirar; } \\
\text { estertores; eupnéico; frequência respiratória; ortopnéia; padrão respiratório; } \\
\text { permeabilidade das vias aéreas; roncos; sibilos; taquipnéia; tosse; uso da } \\
\text { musculatura acessória. }\end{array}$ \\
\hline Hidratação & $\begin{array}{l}\text { Anúria; desidratação; diminuição da umidade das mucosas; diminuição do } \\
\text { turgor e elasticidade da pele; edema; ingestão hídrica; lábios ressecados; } \\
\text { língua seca, oligúria; pele ressecada; polidpsia; poliúria; sede. }\end{array}$ \\
\hline Nutrição & $\begin{array}{l}\text { Alterações dentárias; altura; anemia; anorexia; apetite; avaliação } \\
\text { nutricional; desnutrição; disfagia; dispepsia; engasgo; hábitos alimentares; } \\
\text { IMC- Índice de Massa Corporal; massa corporal; náuseas; obesidade; peso; } \\
\text { perda de peso; perímetro abdominal; pirose; polifagia; regurgitação; } \\
\text { sobrepeso; uso de prótese dentária. }\end{array}$ \\
\hline Eliminação & $\begin{array}{l}\text { Abdômen; constipação; diarréia; diurese espontânea; disúria; dor } \\
\text { abdominal; evacuação; flatulência; hemorroidas; incontinência fecal; } \\
\text { incontinência urinária; nictúria; oligúria; polaciúria; retenção urinária; ruído } \\
\text { hidroaéreo diminuído ou ausente; sonda vesical de demora; uso de fralda; } \\
\text { vômitos. }\end{array}$ \\
\hline Sono e Repouso & $\begin{array}{l}\text { Acorda várias vezes a noite; características do sono; depressão; dorme } \\
\text { durante o dia; uso de medicamentos sedativos. }\end{array}$ \\
\hline $\begin{array}{l}\text { Exercícios e } \\
\text { atividades físicas }\end{array}$ & $\begin{array}{l}\text { Dor ao movimento; exercício físico regular; força muscular; imobilidade; } \\
\text { imobilidade parcial. }\end{array}$ \\
\hline Sexualidade & $\begin{array}{l}\text { Abuso sexual; atividade sexual; dispaurenia; doença sexualmente } \\
\text { transmissível; ressecamento vaginal; uso de preservativo. }\end{array}$ \\
\hline Abrigo & $\begin{array}{l}\text { Casa própria; coleta de lixo; hospitalização; mora sozinho; saneamento } \\
\text { básico. }\end{array}$ \\
\hline Mecânica corporal & $\begin{array}{l}\text { Alterações da marcha; evento de queda; fraturas ósseas; necessidade de } \\
\text { ajuda para deambular. }\end{array}$ \\
\hline Motilidade & $\begin{array}{l}\text { Acamado; amputação de membros; presença de drenos e sondas; uso de } \\
\text { bengalas, andadores e cadeira de rodas. }\end{array}$ \\
\hline
\end{tabular}




\begin{tabular}{|c|c|}
\hline Cuidado corporal & $\begin{array}{l}\text { Capaz de banhar-se; capaz de vestir-se; couro cabeludo; falta de motivação; } \\
\text { frequência de higiene corporal; frequência de higiene bucal; gengivite; grau } \\
\text { de dependência; halitose; higiene das unhas; necessidade de ajuda para } \\
\text { realizar o cuidado; seborreia. }\end{array}$ \\
\hline $\begin{array}{l}\text { Integridade física e } \\
\text { cutaneomucosa }\end{array}$ & $\begin{array}{l}\text { Calos; cicatriz; condições das mucosas; condições da pele; doenças } \\
\text { hematológicas; doenças musculoesqueléticas; eczema; equimoses; } \\
\text { hipocorada; hematomas; história de lesão; icterícia; normocorada; palidez; } \\
\text { pele seca; petéquias; placas senis; prurido; úlceras de decúbito. }\end{array}$ \\
\hline $\begin{array}{l}\text { Regulação } \\
\text { Neurológica }\end{array}$ & $\begin{array}{l}\text { Cefaléia; coordenação dos movimentos; declínio cognitivo; desorientação; } \\
\text { demência; diminuição dos reflexos; dormência; força motora normal; nível } \\
\text { de consciência; orientado no tempo e no espaço; paresia; parestesia; } \\
\text { tremores de extremidades; vertigem. }\end{array}$ \\
\hline Regulação Térmica & $\begin{array}{l}\text { Afebril; calafrios; febre; hipertermia; hipotermia; pele fria; pele quente; } \\
\text { sudorese; temperatura axilar. }\end{array}$ \\
\hline $\begin{array}{l}\text { Regulação } \\
\text { Hormonal }\end{array}$ & $\begin{array}{l}\text { Diabetes; hiperglicemia; hipoglicemia; uso de hipoglicemiantes orais; Uso } \\
\text { de insulina. }\end{array}$ \\
\hline $\begin{array}{l}\text { Regulação } \\
\text { Hidrossalina e } \\
\text { Eletrolítica }\end{array}$ & Débito urinário; pressão arterial; pulso. \\
\hline $\begin{array}{l}\text { Regulação } \\
\text { Imunológica }\end{array}$ & Alergias; cartão de vacina; vacinas. \\
\hline $\begin{array}{l}\text { Crescimento } \\
\text { Celular }\end{array}$ & História familiar de doenças; infertilidade; menopausa. \\
\hline $\begin{array}{l}\text { Regulação } \\
\text { Vascular }\end{array}$ & $\begin{array}{l}\text { Arritmias; ausculta cardíaca; características do pulso; cãimbras; frequência } \\
\text { cardíaca; perfusão periférica; ritmo cardíaco; varizes. }\end{array}$ \\
\hline Locomoção & $\begin{array}{l}\text { Atrofia de membros; deambula; fraqueza muscular; perda de força; } \\
\text { Sequelas motoras. }\end{array}$ \\
\hline $\begin{array}{l}\text { Percepção dos } \\
\text { órgãos dos sentidos }\end{array}$ & $\begin{array}{l}\text { Olfativa: diminuição da percepção olfativa; Visual: acuidade visual } \\
\text { diminuída; alterações visuais; distúrbios da visão; uso de lentes/óculos; } \\
\text { Tátil: sensibilidade à dor; Gustativa: diminuição da sensibilidade } \\
\text { gustativa; Auditiva: acuidade auditiva diminuída; condições da audição; } \\
\text { hipoacusia; uso de prótese auditiva; zumbidos; Dolorosa: artralgia; } \\
\text { expressão facial de dor; mialgia; presença de dor (tipo, local, frequência). }\end{array}$ \\
\hline Ambiente & $\begin{array}{l}\text { Acesso adequado; ambiente livre de perigo; iluminação adequada; renda } \\
\text { familiar. }\end{array}$ \\
\hline Terapêutica & $\begin{array}{l}\text { Exames preventivos; medicações etiquetadas e guardadas em local seguro; } \\
\text { uso de medicamentos. }\end{array}$ \\
\hline \multicolumn{2}{|r|}{ PSICOSSOCIAIS } \\
\hline Segurança & $\begin{array}{l}\text { Calmo; choro; irritabilidade; medo; melancolia; preocupação; visita de } \\
\text { familiares. }\end{array}$ \\
\hline Amor e Aceitação & $\begin{array}{l}\text { Agressividade; angústia; ansiedade; estado conjugal; fuga; insegurança; } \\
\text { negativismo; rejeição; situação de violência; solidão. }\end{array}$ \\
\hline $\begin{array}{l}\text { Liberdade e } \\
\text { Participação }\end{array}$ & $\begin{array}{l}\text { Dependente dos familiares e amigos; independente dos familiares e amigos; } \\
\text { participação no plano terapêutico. }\end{array}$ \\
\hline Comunicação & Afasia; disartria; empatia; gagueira; habilidade para escrever; habilidade \\
\hline
\end{tabular}




\begin{tabular}{|c|l|}
\hline & para ler; uso da linguagem não verbal; uso da linguagem verbal. \\
\hline Criatividade & Habilidades manuais \\
\hline $\begin{array}{c}\text { Aprendizagem } \\
\text { (educação à saúde) }\end{array}$ & $\begin{array}{l}\text { Dúvidas sobre autocuidado; Conhecimento sobre a sua doença; percepção } \\
\text { da saúde; uso de álcool; uso de drogas; uso de tabaco. }\end{array}$ \\
\hline Sociabilidade & Isolamento social; participação em grupos de idosos. \\
\hline Recreação e Lazer & Ocupação do tempo livre; participação em atividades de lazer. \\
\hline $\begin{array}{c}\text { Espaço e Atenção } \\
\text { Autorrealização, } \\
\text { Autoestima e } \\
\text { Autoimagem }\end{array}$ & $\begin{array}{l}\text { Necessidade de atenção; prefere ficar sozinho. } \\
\text { imagem corporal; respeito por si; sentimento de incapacidade; tem medo de } \\
\text { expor ideias. }\end{array}$ \\
\hline \multicolumn{2}{|c|}{ PSICOESPIRITUAIS } \\
\hline $\begin{array}{c}\text { Religiosa ou } \\
\text { teológica, ética ou } \\
\text { de filosofia de vida }\end{array}$ & $\begin{array}{l}\text { Necessidade de atividades religiosas; necessidade de um líder espiritual; } \\
\text { religião. }\end{array}$ \\
\hline
\end{tabular}

Fonte: Dados da pesquisa.

Diante do exposto no Quadro 1, percebe-se que as necessidades psicobiológicas e seus indicadores empíricos se sobressaíram em maior quantidade, seguido das características acerca das necessidades psicossociais e em menor número observou-se os aspectos interligados as necessidades psicoespirituais.

\section{Discussão}

Tendo em vista as particularidades da pessoa idosa, destaca-se a importância de um instrumento que favoreça o processo de comunicação, o raciocínio clínico e a organização das informações do idoso nos mais variados momentos do continuum saúde-doença, viabilizando uma base de dados completa para uma adequada condução da assistência e que, consequentemente, melhore a qualidade do cuidado prestado ao cliente e seus familiares.

À medida que essa assistência é norteada por um referencial teórico, os agentes da Enfermagem tornam-se sujeitos ativos desse processo ao aprimorar habilidades cognitivas e psicomotoras para associar teoria e prática, relacionando conhecimentos multidisciplinares e estabelecendo relações de trabalho mais coerentes e produtivas no sentido de oferecer um cuidado integral e qualificado (Menezes \& Priel, 2011).

Conforme os resultados encontrados, foi evidenciado o predomínio das manifestações das necessidades humanas básicas afetadas na pessoa idosa no nível psicobiológico, com destaque para as necessidades de nutrição, eliminação, integridade física e cutaneomucosa, oxigenação e percepção dos órgãos dos sentidos.

As Necessidades Psicobiológicas são consideradas forças instintivas, como a oxigenação e nutrição. Já as Necessidades Psicossociais são manifestações que ocorrem no indivíduo por meio do nível psicossocial, como realizar trocas sociais, comunicação e lazer. As Necessidades Psicoespirituais podem ser compreendidas como aquelas das quais o homem procura compreender o que vivencia de maneira inexplicável cientificamente, prova disso é a religião. (Souza et al., 2016 \& Santos et al., 2020)

A necessidade de nutrição tem como significado a ingestão, digestão e absorção de nutrientes essenciais à sobrevivência humana (Potter \& Perry, 2013). No idoso, as alterações causadas pelo processo de envelhecimento, acomete várias alterações anatômicas e funcionais, que influencia diretamente na sua saúde e nutrição. Assim, os idosos podem 
experimentar reduções na capacidade funcional, podendo ser observado desde a diminuição do paladar até alterações a nível metabólico (Miranda \& Paiva, 2019).

A necessidade de eliminação é o processo que atende a necessidade do organismo de livrar-se de substâncias indesejáveis ou presentes em quantidades excessivas. Os idosos geralmente têm problemas com excreção sólida e líquida. Constipação é a causa mais comum, sendo consequência da falta de fibras na dieta, ingestão de líquido insuficiente, atividades físicas diminuídas, redução do tônus muscular e ainda o uso abusivo de laxante. A incontinência urinária é também um problema comum para geriatria, por exercer grande impacto na saúde e na qualidade de vida dos idosos, por isso ela deve ser levada a sério, não apenas como sinal de doença, mas como um problema social e higiênico, humilhante e angustiante (Potter \& Perry, 2013).

A necessidade de integridade física e cutaneomucosa é a capacidade do organismo em manter suas características de sensibilidade, elasticidade, vascularização e umidade do tecido epitelial, subcutâneo e mucoso tendo como objetivo a proteção do corpo. Nos idosos, a integridade física e cutaneomucosa pode estar alterada pelos traumas, principalmente como consequência das quedas e agressões. Outras causas de alterações são a imobilidade, as incontinências, má nutrição, estado mental diminuído (Potter \& Perry, 2013).

A necessidade de oxigenação é o processo de utilização do oxigênio nos fenômenos de oxi-redução das atividades vitais (Horta, 2005). Nos idosos, a necessidade de oxigênio se reduz, porque se verifica que todas as estruturas relacionadas com a respiração alteram-se durante o processo de envelhecimento, ocorrendo perda da capacidade de expansão pulmonar, diminuição da capacidade respiratória e aumento da possibilidade de doenças como enfisema e bronquite (Potter \& Perry, 2013).

A necessidade de percepção dos órgãos dos sentidos é manifestada através dos estímulos nervosos, objetivando a interação com o outro e a percepção do ambiente. Nos idosos, as mudanças relacionadas à estimulação sensorial e a mobilidade física ocorrem em graus variáveis, podendo provocar déficits em algumas pessoas dessa faixa etária. Àquelas que apresentam declínios funcionais acentuados tornam-se indivíduos frágeis, passando a depender de terceiros para aa atividades de vida diária, sendo gradualmente mantidos no leito e evoluindo para outras complicações (Freitas \& Py, 2016).

Embora a maioria dos indicadores foi classificado nas necessidades psicobiológicas, destacam-se também as necessidades psicossociais da pessoa idosa como amor, aceitação e comunicação. Já as necessidades psicoespirituais, como religiosa ou teológica, ética ou de filosofia de vida.

Os indivíduos que não têm desfrutam de suas necessidades psicossociais adequadamente podem se sentir sozinhos e afastados dos familiares e amigos. Dessa forma, a comunicação deve ser estimulada no cuidado em saúde aos idosos, subsidiando a atuação do enfermeiro aos pacientes/familiares a um cuidado integral, o que repercute na promoção a saúde mental destes (Pacheco et al., 2020).

A utilização de ferramentas religiosas e espirituais no atendimento de enfermagem é essencial, o respeito a crença religiosa melhora a autoestima dos indivíduos, sendo assim, o aspecto espiritual é considerado um quesito importante no cuidado em saúde aos idosos (Barbosa et al., 2021). Logo, a saúde de um indivíduo depende de um adequado equilíbrio dos fatores físicos, psicológicos, sociológicos, culturais, de desenvolvimento e, certamente, espirituais (Potter \& Perry, 2013).

\section{Conclusão}

O idoso apresenta características específicas, que o diferencia de outros tipos de pacientes. Neste estudo foram levantados indicadores empíricos afetados na pessoa idosa, utilizando como referencial teórico a Teoria das Necessidades Humanas Básicas, de Horta, que subdivide as necessidades em três níveis: psicobiológicas, psicossociais e psicoespirituais. Foi 
realizado uma revisão na literatura, onde foi visto cada necessidade e identificado para cada uma delas seus indicadores empíricos.

O estudo permitiu o alcance dos objetivos proposto em dois momentos. Em primeiro plano, foi realizada uma revisão integrativa da literatura sobre as necessidades humanas básicas da pessoa idosa, sendo feito um amplo amparato bibliográfico. Nesta etapa foram identificados 316 indicadores empíricos, sendo 243 indicadores das necessidades psicobiológicas, 67 pertencentes às necessidades psicossociais e 6 às necessidades psicoespirituais. A segunda etapa compreendeu a validação dos indicadores empíricos oriundos da primeira etapa. Do total de 316 indicadores empíricos apontados na literatura e analisados pelos enfermeiros, 255 permaneceram com IVC $\geq 0.80$ e, desses indicadores validados, 205 foram das necessidades psicobiológicas, 47 das necessidades psicossociais e 3 das necessidades psicoespirituais.

Nesse sentido, conclui-se que é possível elaborar um instrumento de avaliação da condição de saúde da pessoa idosa na atenção básica a partir dos indicadores empíricos mencionados e validados por um grupo de especialistas. Acredita-se que os achados dessa pesquisa venham a contribuir com a implantação da sistematização da assistência de enfermagem na atenção básica. Por outro lado, por meio de um instrumento específico, os enfermeiros que atuam nas Unidades de Saúde da Família se interessem pela SAE, tendo a percepção de que essa metodologia facilitará o trabalho, aumentando a interação enfermeiropaciente, afim de melhorar a qualidade da assistência prestada e proporcionar a autonomia da categoria profissional.

A realização dessa pesquisa favoreceu uma base teórica em relação ao cuidado de enfermagem com a pessoa idosa, assim como, também possibilitou uma melhor compreensão da Teoria das Necessidades Humanas Básicas através da inclusão de novos conhecimentos na temática referida. Sendo assim, sugere-se a continuidade da propagação do saber científico através de novos estudos e publicações acerca da temática referida em outros municípios e regiões do Brasil.

\section{Referências}

Alexandre, N. M. C., \& Coluci, M. Z. O. (2011). Validade de conteúdo nos processos de construção e adaptação de instrumentos de medidas. Ciência \& Saúde Coletiva, 16(7), 3061-3068. https://dx.doi.org/10.1590/S1413-81232011000800006

Barbosa, D. J. et al. (2021). Where religions meet: a study of social representations of psychoactive drugs and their implications for nursing care. Research, Society and Development, 10(1), e17310110982. https://doi.org/10.33448/rsd-v10i1.10982

Brasil (1986). Lei $n^{\circ} 7.498 / 86$, de 25 de junho de 1986. Lei do Exercício Profissional da Enfermagem, Brasília (DF). http://www.cofen.gov.br/lei-n-749886de-25-de-junho-de-1986_4161.html

Conselho Federal de Enfermagem (1993). Resolução COFEN-159/1993. Dispõe sobre a consulta de enfermagem. Rio de Janeiro (Brasil): COFEN. http://www.cofen.gov.br/resoluo-cofen-1591993_4241.html.

Cheloni, I. G., Silva, J. V. S., \& Souza, C. C. (2020). Necessidades humanas básicas afetadas em pacientes oncológicos: revisão integrativa da literatura. HU Revista, 46, 1-11. https://doi.org/10.34019/1982-8047.2020.v46.29242

Fawcett, J. (2013). Thoughts about conceptual models and measurement validity. Nurs Sci Q. 26(2):189-91. 10.1177/0894318413477143.

Ferreira, A. de S., \& Abrahão, A. L. (2020). Nursing care management in the Family Health Strategy: systematic review. Research, Society and Development, 9(5), e68953087. https://doi.org/10.33448/rsd-v9i5.3087

Freitas, E.V. \& Py, L. (2016). Tratado de geriatria e gerontologia. (4a ed.), Guanabara Koogan.

Horta, W. A. (2005). Processo de enfermagem. Editora Pedagógica Universitária. EPU.

Jacon, J. C. et al. (2020). Identificação de diagnósticos de enfermagem em nefropatas em hemodiálise à luz da teoria das necessidades humanas básicas. CuidArte, Enferm, p. 48-54. https://pesquisa.bvsalud.org/portal/resource/pt/biblio-1119288

Marques, D. K. A, Moreira, G. A. C., \& Nóbrega, M. M. L (2008). Análise da teoria das necessidades humanas básicas de Horta. Rev. Enferm UFPE On line; 2(4):410-16.

Martins, J. D. N. et al. (2020). Nursing care for the control of disabilities in elderly diabetics in primary health care. Research, Society and Development, 9(8), e686985915. https://doi.org/10.33448/rsd-v9i8.5915

Menezes, S. R. T., Priel, M. R. \& Pereira, L. L. (2011). Autonomia e vulnerabilidade do enfermeiro na prática da Sistematização da Assistência de Enfermagem. Rev Esc Enferm. 45(4):953-8. 
Research, Society and Development, v. 10, n. 3, e9810313077, 2021

(CC BY 4.0) | ISSN 2525-3409 | DOI: http://dx.doi.org/10.33448/rsd-v10i3.13077

Miranda, R. N. A. \& Paiva, M. B. (2019). Antropometria e consumo alimentar: identificador do estado nutricional de idosos. Nutrição Brasil, 18(3), 141-150. https://doi.org/10.33233/nb.v18i3.2839

Pacheco, L. S. P. et al. (2020). The nurse's effective communication process with the patient in palliative care. Research, Society and Development, 9(8), e747986524. https://doi.org/10.33448/rsd-v9i8.6524

Pereira, A. S., et al. (2018). Metodologia da pesquisa científica. UFSM. https://repositorio.ufsm.br/bitstream/handle/1/15824/Lic_Computacao_MetodologiaPesquisa-Cientifica.pdf?sequence $=1$.

Piccinini, V., Costa, A., \& Pissaia, L. (2018). Implantação da Sistematização da Assistência de Enfermagem como meio de qualificação da assistência ao idoso. Revista Brasileira De Ciências Do Envelhecimento Humano, 14(3). https://doi.org/10.5335/rbceh.v14i3.6631

Pinto et al. (2017). Conceito de ser humano nas teorias de enfermagem: aproximação com o ensino da condição humana. Pro-Posições, 28(Suppl. 1), 88110. https://dx.doi.org/10.1590/1980-6248-2015-0164

Potter, P.A., Perry, A.G (2013). Fundamentos de enfermagem. (8a ed.), Elsevier.

Santos, B. R. F. et al. (2020). Sistematização da assistência de enfermagem em isolamento respiratório pediátrico a fulgor da teoria das necessidades humanas básicas: relato de experiência. Revista Eletrônica Acervo Saúde, (50), e2914-e2914. https://doi.org/10.25248/reas.e2914.2020

Silva, L. F., Russo, R. D. F. S. M., \& Oliveira, P. S. G. (2018). Quantitativa ou qualitativa? um alinhamento entre pesquisa, pesquisador e achados em pesquisas sociais. Revista Pretexto, 30-45. https://doi.org/10.21714/pretexto.v19i4.5647

Silva, P.C. (2014). Implantação da Sistematização da Assistência de Enfermagem (SAE) em um CAPS: um estudo de caso. Monografia de Especialização em Linhas de Cuidado em Enfermagem. Departamento de Enfermagem, Universidade Federal de Santa Catarina. 31p.

Siqueira, P. L. F. (2020). Systematization of assistance, theories and nursing process - a literature review. Research, Society and Development, 9(10), e4419108667. https://doi.org/10.33448/rsd-v9i10.8667

Souza, T.L. et al. (2016). Necessidades humanas básicas alteradas em pacientes pós transplante renal: estudo transversa. Online Brazlian Jounal of Nurse; 15 (2):265-275. https://doi.org/10.17665/1676-4285.20165253 